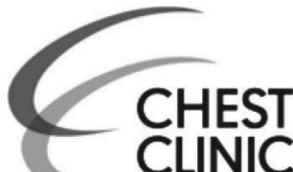
CASE BASED DISCUSSION

\section{A lesson in plasticity: a 74-year-old man with plastic bronchitis}

\author{
Anand Shah, ${ }^{1,2}$ Jackie Donovan, ${ }^{3}$ Phil Marino, ${ }^{4}$ Pallav L Shah, ${ }^{1,2}$ Anand Devaraj, ${ }_{1}^{2,5}$ \\ Kshama Wechalekar, ${ }^{2,6}$ Toby M Maher, ${ }^{2,7}$ Michael R Loebinger, ${ }^{1,2}$ Robert Wilson ${ }^{1,2}$
}

${ }^{1}$ Host Defence Unit, Royal Brompton and Harefield NHS Foundation Trust, London, UK ${ }^{2}$ Imperial College London, London, UK

${ }^{3}$ Department of Biochemistry, Royal Brompton and Harefield NHS Foundation Trust, London, UK

${ }^{4}$ Lane Fox Respiratory Unit, Guy's and St Thomas' NHS Foundation Trust, London, UK ${ }^{5}$ Department of Radiology, Royal Brompton and Harefield NHS Foundation Trust, London, UK ${ }^{6}$ Nuclear Medicine, Royal Brompton and Harefield NHS Foundation Trust, London, UK ${ }^{7}$ Interstitial Lung Disease Unit, Royal Brompton and Harefield NHS Foundation Trust, London, UK

\section{Correspondence to}

Dr Anand Shah, Host Defence Unit, Royal Brompton and Harefield NHS Foundation Trust, Sydney Street, London SW3 6NP UK; s.anand@imperial.ac.uk

Received 8 January 2017 Revised 6 March 2017 Accepted 7 March 2017 Published Online First 3 April 2017

\section{CrossMark}

To cite: Shah A, Donovan J, Marino $\mathrm{P}$, et al. Thorax 2017;72:1055-1057.
AS: A 74-year-old male was transferred to the Royal Brompton hospital from a local hospital with respiratory failure and expectoration of bronchial casts (see figure 1A). The patient recalls recurrent chest infections with occasional haemoptysis in his teens with no prior history of asthma, TB or pertussis. Despite investigation and antibiotic treatment, his symptoms of intermittent, chronic recurrent bronchial cast expectoration continued. A fibreoptic bronchoscopy on the patient, aged 41, revealed erythematous bronchial mucosa in the right middle lobe, with a ventilation/perfusion scan revealing concomitant decreased ventilation. Bronchial washings were unremarkable, and given concern of a pathology originating from the right middle lobe, a right middle lobectomy was performed. Histology showed non-specific inflammatory fibrosis around the lobar bronchus.

His symptoms, however, did not resolve. Microbiological examination of his casts revealed no growth, and histological examination showed fibrin and mucus with some lymphocytes. Further trials of corticosteroids, nebulised $\mathrm{N}$-acetylcysteine, hypertonic saline, prophylactic azithromycin, systemic antifungals and oral cyclophosphamide were unsuccessful. Extensive immunological and allergic tests were normal.

His symptoms remained stable till age 73 when cast expectoration and infections grew more frequent. Recently, his condition had deteriorated, requiring admission locally with respiratory failure. $\mathrm{He}$ was transferred to our centre for further evaluation.

On arrival, the patient was in respiratory distress. On a $\mathrm{FiO}_{2}$ of $60 \%$, oxygen saturations were $90 \%$ with arterial blood gas analysis showing a $\mathrm{pO}_{2}$ of $6.72 \mathrm{kPa}, \mathrm{pCO}_{2}$ of $6.31 \mathrm{kPa}, \mathrm{pH}$ of 7.38 and bicarbonate of $30.5 \mathrm{mmol} / \mathrm{L}$. A chest radiograph showed significant right-sided consolidation. Blood tests revealed a normal white cell count with a $\mathrm{C}$ reactive protein of $10 \mathrm{mg} / \mathrm{L}$. He was empirically started on intravenous tazocin, caspofungin, hydrocortisone, crystalloid fluids and nebulised bronchodilators, and transferred to a high-dependency unit. Evaluation did not reveal yellow nails or history of sinusitis. Further enquiry revealed coexistent ischaemic heart disease with previous stents to his left anterior and posterior descending coronary arteries. He was a 5 pack-year ex-smoker and lived with family with no environmental exposures of note. Lung function preadmission showed a forced expiratory volume of $1.05 \mathrm{~L} / \mathrm{s}$, a forced vital capacity of $2.37 \mathrm{~L}$ and an FEV1:FVC of 0.44. A high-resolution CT scan was performed (figure $1 \mathrm{~B}, \mathrm{C}$ ).

$A D$ : CT imaging demonstrated marked diffuse bronchial wall thickening with mild bilateral patchy ground glass opacities with extensive consolidation in the right upper lobe (figure 1B, C). Minimal interlobular septal thickening in the lower lobes was noted.

$A S, M L$ and $R W$ : This patient presented with a long history of 'plastic bronchitis'. Plastic bronchitis is a rare but well described disease with formation of firm gelatinous mucous casts causing bronchial obstruction. Historically, plastic bronchitis was classified histologically as either inflammatory' type I casts, which are cellular with abundant fibrin, eosinophils and Charcot-Leyden crystals, or 'non-inflammatory' type II casts, which are acellular with mucin and little fibrin. The aetiology remains unknown, but is most commonly associated with lymphatic abnormalities with expectoration of chylous casts. Other associated conditions include cyanotic congenital heart disease, cystic fibrosis and sickle cell disease.

In the above case, the underlying aetiology and classification of plastic bronchitis had not been established. Unfortunately, histology was unavailable from his previous lobectomy, eliminating the possibility of staining with novel lymphatic markers (eg, D2-40, LYVE-1). We therefore focused our attention on examination of the patient's bronchial casts.

JD: Histological examination of the casts showed a relatively acellular cast with mucin and fibrin. Microbiological examination was unrevealing. Centrifugation after liquefaction of his casts (by vortexing in phosphate-buffered saline and centrifugation at $1000 \mathrm{~g}$ for $10 \mathrm{~min}$ ) however revealed a turbid fatty layer, with biochemical analysis confirming a chylous nature with a triglyceride level of $32.5 \mathrm{mmol} / \mathrm{L}$ (figure 1D).

$A S, M L$ and $R W:$ We commenced treatment with a low fat, medium-chain triglyceride diet and octreotide, a somatostatin analogue. It is proposed that octreotide causes mild vasoconstriction of splanchnic vessels, including hepatic venous flow. This leads to reduction in gastric, pancreatic and intestinal secretions as well as intestinal absorption collectively reducing the flow of chyle. The patient, however, had significant bronchial casts, and a means of either removal or liquefaction was required. A flexible bronchoscopy approach was considered.

PS: Removal of bronchial casts by bronchoscopy is difficult. The casts are often too firm for removal 

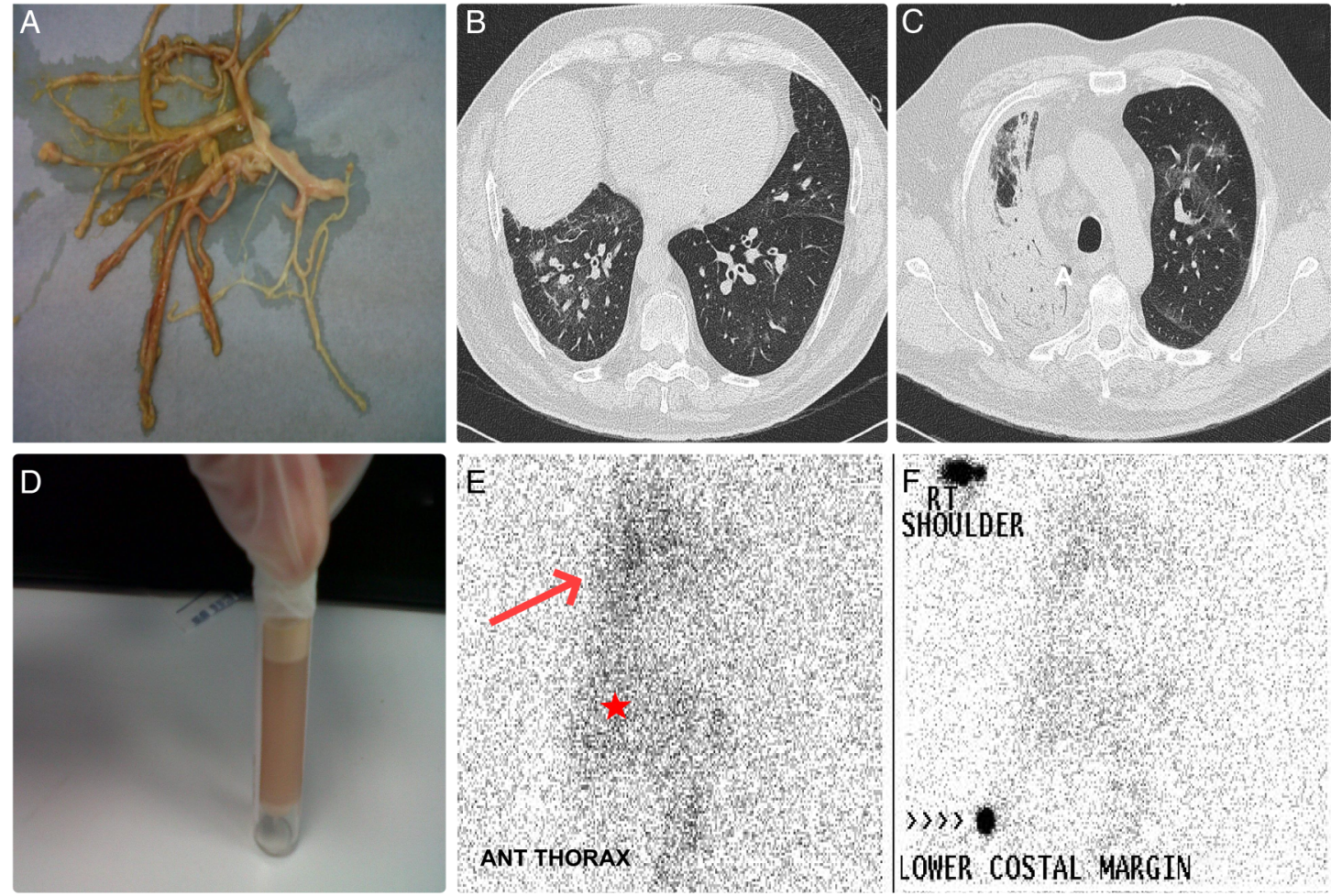

Figure 1 (A) Representative image of an expectorated bronchial cast by the patient. (B and C) CT image demonstrating marked diffuse bronchial wall thickening with mild bilateral patchy ground glass opacities with extensive consolidation in the right upper lobe. Minimal interlobular septal thickening in the lower lobes is also noted. (D) Image following centrifugation of a liquefied bronchial cast expectorated by the patient revealing a turbid fatty layer confirming its chylous nature. (E) Images of lymphangioscintogram (anterior thorax with and without right shoulder and costal margin markers) showing abnormal tracer activity in the right lung (indicated by red arrow) confirming chylous leak. Tracer activity is also seen in the abdominal lymphatic channels and in the liver (indicated by red star) due to systemic circulation of radiotracer on delayed images.

by suction, yet too friable for removal by forceps. Novel technology such as cryotherapy may be useful, and hence bronchoscopy was attempted. No bronchial casts were visible, however, with oedematous collapsed right lower and upper lobe bronchi. No therapeutic procedures were performed. Microbiology was unrevealing, and bronchial biopsies showed non-specific inflammation.

$A S, M L$ and RW: We hence focused on cast liquefaction. The use of both nebulised dornase- $\alpha$ (DNase) and tissue plasminogen activator ( $t-P A)$ has been reported for the management of chylous bronchial casts. ${ }^{2}$ Due to coexistent dual antiplatelet therapy following drug-eluting coronary arterial stent implantation, we initially examined in vitro efficacy.

$J D$ : A dried bronchial cast expectorated by the patient was incubated for 2 hours in $2 \mathrm{~mL}$ of $0.1 \mathrm{mg} / \mathrm{mL} \mathrm{t}-\mathrm{PA}, 0.1 \mathrm{mg} / \mathrm{mL}$ DNAse or saline. The cast was removed, air-dried and reweighed, with supernatant analysis for protein and fibrin breakdown products. Incubation with t-PA resulted in structure loss, $62 \%$ cast mass reduction, $0.47 \mathrm{~g} / \mathrm{L}$ protein and $>4 \times 10^{6} \mathrm{ng} / \mathrm{mL}$ soluble cross-linked fibrin (XDP) release. DNAase incubation resulted in no structural change, $10 \%$ mass reduction, $0.07 \mathrm{~g} / \mathrm{L}$ protein and $40000 \mathrm{ng} / \mathrm{mL}$ XDP release. Saline incubation resulted in no structural change, $2 \%$ mass reduction, $0.0 \mathrm{~g} / \mathrm{L}$ protein and $1200 \mathrm{ng} / \mathrm{mL}$ XDP release.

$A S, M L$ and RW: Biochemical analysis showed partial and high liquefaction efficacy of DNAse and t-PA, respectively. Within a high-dependency environment, nebulised DNase $2.5 \mathrm{mg}$, two times per day, and t-PA $5 \mathrm{mg}$, three times per day, were started. No haemorrhagic complications were seen with radiological improvement within 24 hours. However, liquefaction led to difficulty in clearance, intermittent hypoxia and cast reformation with further bronchial obstruction. We therefore focused on understanding the pathophysiology of chylous bronchial cast formation.

PM: Post Fontan procedure due to congenital heart disease, it is thought that high central and pulmonary venous pressures may play an aetiological role in cast formation. Hence, an echocardiogram was performed. This showed normal left ventricular systolic and diastolic function with mild hypokinesia of the posterior inferior wall. The right heart was mildly dilated with normal longitudinal function. There was no significant valvular disease, normal-sized right atrium and an estimated pulmonary arterial systolic pressure of $51 \mathrm{~mm} \mathrm{Hg}$. Given evidence of right heart strain on echocardiogram and moderately elevated pulmonary pressures, the patient was started on pulmonary vasodilator therapy with sildenafil and uptitrated to $50 \mathrm{mg}$ three times per day.

$A S, M L$ and RW: There have been recent reports regarding the use of the mammalian target of rapamycin (mTOR) inhibitor sirolimus (rapamycin) for management of recurrent chylothoraces in patients with lymphangioleiomyomatosis. ${ }^{3}$

TM: Lymphangioleiomyomatosis is a progressive cystic lung disease in women associated with inappropriate activation of the mTOR signalling pathway causing dysregulation of cellular growth and lymphangiogenesis. This can result in recurrent chylothoraces and, on rare occasions, chyloptysis. Recent studies have highlighted the successful use of sirolimus in the management of chylothoraces and stabilisation of lung function. The mechanism of action is unclear, but potentially relates to the regression of abnormal smooth muscle cellular proliferation 
causing obstruction and leak within the lymphatic system. In the current case, the reported symptoms since childhood may point to a diagnosis of generalised lymphatic anomaly.

Generalised lymphatic anomaly is a group of disorders that can include conditions with congenital, benign, focal areas of lymphatic proliferation occurring in any part of the body containing lymphatics. This pathological process can result in restrictive and/or obstructive physiology, chylous effusions and respiratory failure and is predominantly detected in children and young adults.

$A S, M L$ and RW: Given the likelihood of lymphatic obstruction alongside clinical deterioration, we started treatment with sirolimus aiming for a therapeutic range of $5-10 \mathrm{ng} / \mathrm{mL}$. Gradually, the patient improved and was discharged from hospital. Subsequent lung function and CT imaging revealed almost complete resolution of his right-sided lung changes and markedly improved lung function. To confirm lymphatic obstruction, a lymphoscintogram was performed (figure 1E). This showed abnormal activity predominantly in the right lung, confirming chylous leak. Recently described novel magnetic resonance lymphangiography techniques potentially enable improved imaging and therapeutic percutaneous embolisation of thoracic lymphatic leak. ${ }^{4}$ This expertise however is limited and was unavailable locally at the time of the patient's presentation.

Three months after discharge, the patient no longer expectorated bronchial casts and continued on sirolimus, one time per day DNase and a low-fat medium chain triglyceride (MCT) diet. Two years later, only rare small episodes of chylous bronchial cast expectoration have occurred with a dramatic improvement in quality of life and respiratory function. No significant side effects from sirolimus therapy have been noted.

AS: Sirolimus is a promising therapeutic option in the management of patients with lymphatic obstruction or chylous leak. It is a macrolide structurally related to the calcineurin inhibitor FK506 and has potent immunosuppressive properties. Sirolimus binds with and inhibits mTOR, a serine/threonine kinase regulated by phosphoinositide-3-kinase. This inhibits the mTOR-mediated signal transduction pathways, resulting in the arrest of cell cycle in G1 phase in various cell types. Inhibition of interleukin 2-induced proliferation of T cells also makes it a valuable immunosuppressant to prevent organ rejection in solid-organ transplantation. Recent genetic studies in patients with isolated and syndromic lymphatic malformations, however, have shown somatic mutations in the catalytic subunit of phosphoinositide-3-kinase (PIK3CA) causing overproliferation of lymphatic endothelial cells with improvement on sirolimus therapy with antilymphangiogenic effects. ${ }^{5}$ This finding may explain the underlying mechanism of action, and importantly, provide a future target for gene therapy in congenital lymphatic obstruction.

Contributors All authors were involved in the clinical care of the patient in this case. JD provided biochemical analysis. AD and KW provided radiological input. AS drafted the manuscript, and all authors assisted in the editorial process.

Competing interests None declared.

Patient consent Obtained.

Provenance and peer review Not commissioned; externally peer reviewed.

Data sharing statement Any data or images from this publication are available, if required, to readers via email.

\section{REFERENCES}

1 Brogan TV, Finn LS, Pyskaty DJ Jr, et al. Plastic bronchitis in children: a case series and review of the medical literature. Pediatr Pulmonol 2002;34:482-7.

2 Lubcke NL, Nussbaum VM, Schroth M. Use of aerosolized tissue plasminogen activator in the treatment of plastic bronchitis. Ann Pharmacother 2013;47:e13.

3 Moua T, Olson EJ, Jean $\mathrm{HC}$, et al. Resolution of chylous pulmonary congestion and respiratory failure in lymphangioleiomyomatosis with sirolimus therapy. Am J Respir Crit Care Med 2012;186:389-90.

4 Itkin MG, McCormack FX, Dori Y. Diagnosis and Treatment of lymphatic plastic bronchitis in adults using advanced lymphatic imaging and percutaneous embolization. Ann Am Thorac Soc 2016;13:1689-96.

5 Boscolo E, Coma S, Luks VL, et al. AKT hyper-phosphorylation associated with PI3K mutations in lymphatic endothelial cells from a patient with lymphatic malformation. Angiogenesis 2015;18:151-62. 